\title{
Band 3, an essential red blood cell hub of activity
}

\section{Timothy J. Satchwell ${ }^{1,2,3}$ and Ashley M. Toye ${ }^{1,2,3}$}

${ }^{1}$ School of Biochemistry, University of Bristol; ${ }^{2}$ National Institute for Health Research (NIHR) Blood and Transplant Research Unit in Red Blood Cell Products, University of Bristol and ${ }^{3}$ Bristol Institute of Transfusion Sciences, NHSBT Filton, Bristol, UK.

E-mail: ASHLEYTOYE - Ash.M.Toye@bristol.ac.uk

doi:10.3324/haematol.2021.278643

T he red blood cell (RBC) is a marvel of cellular evolutionary specialization. Whilst often considered simplistic owing to its absence of nuclei and other cellular organelles, this inability to respond transcriptionally and to replenish components through new protein synthesis necessitates complex post-translational mechanisms through which the cell is able to control, adapt and regulate its key functions in the different environments it experiences traversing the circulation.

In this context, the membrane of the erythrocyte, perhaps more than any other cell, plays a crucial role. Erythrocyte membranes have a unique structure, comprising an array of integral membrane proteins with varied antigenic, transport and mechanical functions arranged in diverse multiprotein complexes. ${ }^{1}$ Underlying the membrane and connected to multicomplexes via adaptor proteins is the spectrin-based cytoskeletal meshwork. These protein interactions together with the membrane lipids themselves impart the characteristic properties of the biconcave RBC.

Central to the stability and functional regulation of the RBC membrane is band 3 (Anion exchanger 1, AE1), its most abundant protein at approximately $1.2 \times 10^{6}$ copies per cell. Its unassuming name (a relic of its original identification as the third band on a Coomassie-stained sodium dodecyl sulfate polyacrylamide gel electrophoresis separation of RBC membrane 'ghosts') belies the importance this protein plays not just through its direct contribution to gas exchange through the electroneutral exchange of chloride ions with the bicarbonate product $\left(\mathrm{HCO}_{3}^{-}\right)$of carbon dioxide metabolism, but as the major site of membrane cytoskeletal connectivity and a hub for hemoglobin and glycolytic enzyme binding.

A substantial body of evidence supports the binding of glyceraldehyde 3-phosphate dehydrogenase (GAPDH) to the cytosolic $\mathrm{N}$-terminus of band $3^{3,4}$ with competitive binding with the deoxygenated form of hemoglobin, first proposed by the Low group, to direct the metabolism of glucose between glycolysis and the pentose phosphate pathway in response to cellular needs and the requirement for antioxidative measures. Put simplistically, band 3 and its interactions act as the RBC molecular switch to sense the oxygenated/deoxygenated state, imparting an appropriate metabolic response (see summary schematic in Figure 1A, B).

Crammed into a single paper published in this issue of Haematologica, Issaian and colleagues have completed a heroic effort to deepen our understanding of the role that glycolytic enzyme binding to band 3 plays in erythrocyte
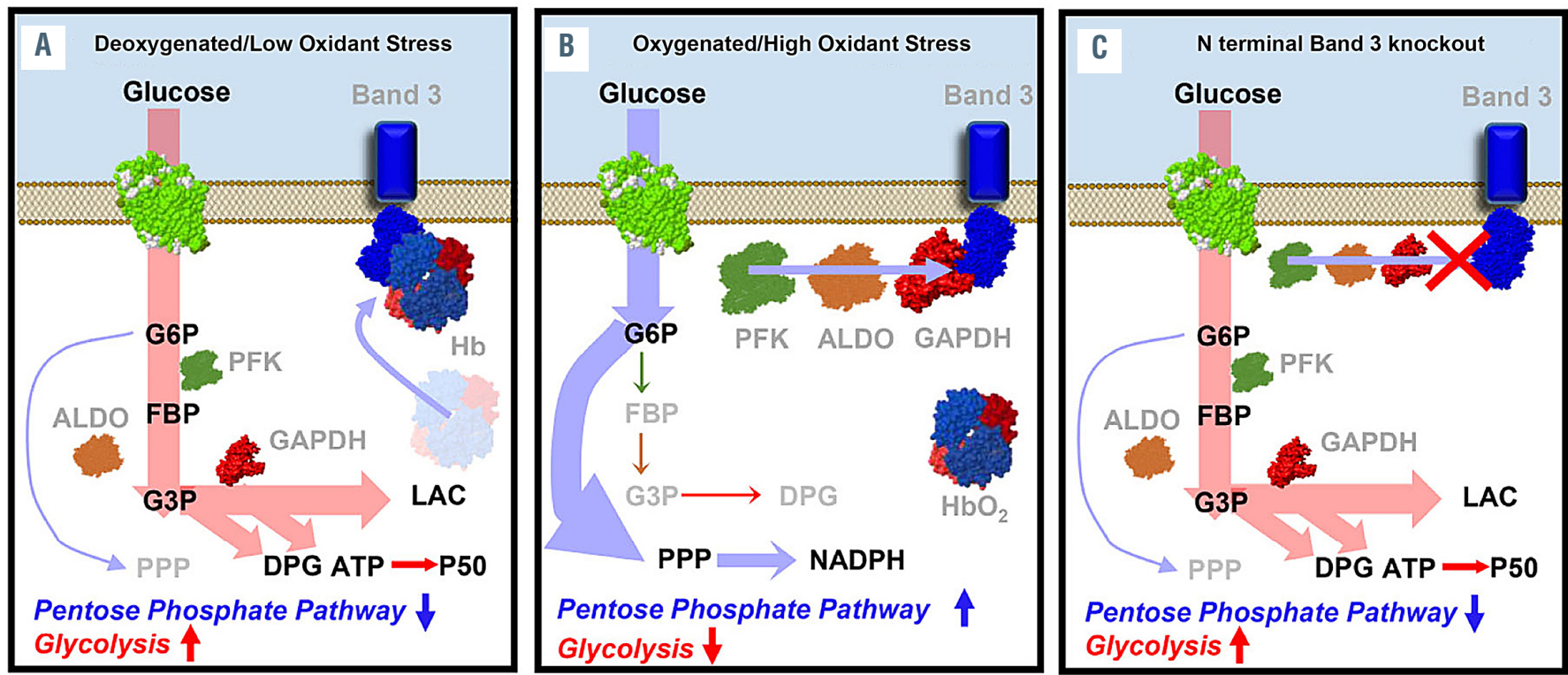

Figure 1. The influence of band 3 on red blood cell metabolism in different conditions. The schematic, adapted from Issaian et al. ${ }^{5}$ shows different conditions and the influence of band 3 on red blood cell metabolism in three different scenarios. (A) In deoxygenation/low oxidant stress conditions, deoxyhemoglobin can bind to the $\mathrm{N}$-terminus of band 3, while glycolytic enzymes become displaced from the same region and are then activated, resulting in increased fluxes through glycolysis and decreased fluxes through the pentose phosphate pathway. (B) In oxygenated or high oxidative stress conditions, glycolytic enzymes can bind to the N-terminus of band 3 , resulting in their partial inhibition, decreasing the metabolic flux through glycolysis and increasing flux through the pentose phosphate pathway to generate the reducing equivalent NADPH necessary to counteract oxidant stress. (C) In the absence of the extreme $\mathrm{N}$-terminus of band 3 during storage, under high oxidative stress conditions, glycolytic enzymes cannot bind band $3 \mathrm{~N}$-termini, so glyceraldehyde 3-phosphate dehydrogenase flux continues, and the pentose phosphate pathway is suppressed, leading to enhanced oxidative stress. G6P: glucose-6-phosphate; PFK: phospho-fructokinase; FBP: fructose 1,6, biphosphatase; ALDO: aldolase; G3P: glucose-3-phosphate; GAPDH: glyceraldehyde 3-phosphate dehydrogenase; DPG: diphosphoglycerate; ATP: adenosine triphosphate; LAC: lactate; Hb: hemoglobin; PPP: pentose phosphate pathway: NADPH: nicotinamide adenine dinucleotide phosphate. Image adapted from Issaian et al. ${ }^{5}$ 
metabolism. ${ }^{5}$ This was achieved using erythrocytes from well-characterized mouse models and a rare natural human variant (Band 3 Neapolis) with truncations at the band $3 \mathrm{~N}$-terminus, in combination with detailed metabolic analyses. The authors demonstrate that loss of band 3 or mutation of the $\mathrm{N}$-terminal GAPDH binding site causes a failure of pentose phosphate pathway activation, with concurrent defects in glutathione recycling and evidence of increasing oxidation products arising during storage in the absence of the extreme $\mathrm{N}$-terminal 11 amino acids of band 3 (summarized in Figure 1C). Interestingly, the authors also show that reintroduction of the band $3 \mathrm{~N}$ terminus using cell-penetrating peptides can rescue the observed metabolic defects. This metabolomics work is striking in the new level of detail it provides about the metabolism of normal and variant RBC.

Another impressive feature of this manuscript is the depth of characterization of the band $3 \mathrm{~N}$-terminal interactome provided by the authors. Varied proteomic approaches, including chemical crosslinking, characterized site-specific interaction interfaces between GAPDH and band 3 , with additional prospective interacting proteins reported including enzymes involved in glutathione synthesis, recycling and lipid peroxidation pathways. However, dissecting the functional relevance of the band 3 interactome is beset with substantial challenges, with its own abundance, existence within at least three (and potentially many more sub-) populations each with genuine direct and additional indirect interactants, ${ }^{6}$ all complicating interpretation. It is unlikely and indeed in some cases impossible (where binding sites for multiple proteins overlap) that all of the proteins reported bind to all of the copies of band 3 , or even every band 3-containing complex. Efforts such as this, and others to uncover not just the identity but the plasticity and relevance of such interactions under physiologically relevant conditions, be they altered oxygenation, ${ }^{7}$ tonicity, ${ }^{8}$ mechanical deformation $^{9}$ or storage such as the work described here, are key to our evolving understanding of RBC structure-function relationships.

Finally, given the essential role band 3 plays in RBC metabolism, the manuscript also touches on the potential of band 3 polymorphisms to influence storage characteristics, using data from the REDSIII storage study. ${ }^{10}$ Here a note of caution needs to be applied as, besides a handful of already known polymorphisms in the band $3 \mathrm{~N}$-terminus (previously shown to be associated with hereditary spherocytosis), it remains unclear what precise impact these mutations have. Any alteration in band 3 abundance has knock-on effects on RBC membrane stability which will impact on the expression of band 3-dependent and -independent proteins alike. Nevertheless, more widely, further determination of the mechanistic basis by which polymorphisms (both pathogenic and non-pathogenic) can influence properties of the RBC beyond those most obviously apparent represents an important continuing endeavor.

\section{Disclosures}

No conflicts of interest to disclose.

\section{Contributions}

TJS and AMT wrote the review together. The views expressed are those of the authors and not necessarily those of the National Health Service, the NIHR or the Department of Health and Social Care.

\section{Funding}

TJS and the work in AMT's laboratory is funded in part by a National Institute for Health Research (NIHR) Blood and Transplant Research Unit in Red Blood Cell Products award (IS-BTU-1214-10032) (University of Bristol) and also National Health Service Blood and Transplant (NHSBT) R\&D grants (WP15-04; WP15-05).

\section{References}

1. Mankelow TJ, Satchwell TJ, Burton NM. Refined views of multiprotein complexes in the erythrocyte membrane. Blood Cells Mol Dis. 2012;49(1):1-10

2. Fairbanks G, Steck TL, Wallach DF. Electrophoretic analysis of the major polypeptides of the human erythrocyte membrane. Biochemistry. 1971;10(13):2606-2617.

3. Chu H, Low PS. Mapping of glycolytic enzyme-binding sites on human erythrocyte band 3. Biochem J. 2006;400(1):143-151.

4. Lewis IA, Campanella ME, Markley JL, Low PS. Role of band 3 in regulating metabolic flux of red blood cells. Proc Natl Acad Sci U S A. 2009;106(44):18515-18520.

5. Issaian A, Hay A, Dzieciatkowska $M$, et al. The interactome of the $\mathrm{N}$-terminus of band 3 regulates red blood cell metabolism and storage quality. Haematologica. 2021;106(11):2971-2985.

6. van den Akker E, Satchwell TJ, Williamson RC, Toye AM. Band 3 multiprotein complexes in the red cell membrane; of mice and men. Blood Cells Mol Dis. 2010;45(1):1-8.

7. Chu H, McKenna MM, Krump NA, et al. Reversible binding of hemoglobin to band 3 constitutes the molecular switch that mediates $\mathrm{O} 2$ regulation of erythrocyte properties. Blood. 2016;128(23): 2708-2716.

8. Hsu K, Lee TY, Periasamy A, et al. Adaptable interaction between aquaporin- 1 and band 3 reveals a potential role of water channel in blood CO2 transport. FASEB J. 2017;31(10):4256-4264.

9. Anong WA, Weis TL, Low PS. Rate of rupture and reattachment of the band 3 -ankyrin bridge on the human erythrocyte membrane. J Biol Chem. 2006;281(31):22360-22366.

10. Kanias T, Lanteri MC, Page GP, et al. Ethnicity, sex, and age are determinants of red blood cell storage and stress hemolysis: results of the REDS-III RBC-Omics study. Blood Adv. 2017;1(15):1132-1141. 